Cytogenet Genome Res 1979;24:257

\title{
Human Genetic Mutant Cell Repository Index
}

A (3;17) balanced translocation, 46 chromosomes

(GM-2808): 252 A (7;18) translocation, 46, XY (GM-657): 126 A 13;13 tandem chromosome translocation in a

subject with congenital anomalies (GM-2018):

$72 \mathrm{~A}(\mathrm{Y} ; 17)$ translocation in a fibroblast culture from

a female with 46 chromosomes (GM-2598): 198 A derivative chromosome 13pat in a subject with

multiple congenital abnormalities (GM-1224):

197

Duplication of a segment of chromosome 3 in a subject with multiple congenital anomalies and a 47, XYY father, inversion of chromosomes 3 and 9 in the mother, and inversion of chromosome 9 in a brother (GM-1253, GM-1252, and GM-1251): 127

Lesch-Nyhan syndrome (GM-2290, 2291, 2292, 2338, 3115, 3116, and 3117): 199 